\title{
Adsorption geometry and electronic properties of flat-lying monolayers of tetracene on the $\operatorname{Ag}(111)$ surface
}

\author{
N. L. Zaitsev, ${ }^{1, *}$ I. A. Nechaev, ${ }^{2,3,4}$ U. Höfer, ${ }^{5,6}$ and E. V. Chulkov ${ }^{2,3,4,6,7}$ \\ ${ }^{1}$ Department of Chemistry, Philipps-Universität Marburg, D-35032, Marburg, Germany \\ ${ }^{2}$ Centro de Física de Materiales CFM-MPC, Centro Mixto CSIC-UPV/EHU, 20018, San Sebastián, Spain \\ ${ }^{3}$ Tomsk State University, Laboratory of Nanostructured Surfaces and Coatings, 634050, Tomsk, Russia \\ ${ }^{4}$ Saint Petersburg State University, Laboratory of Electronic and Spin Structure of Nanosystems, 198504, Saint Petersburg, Russia \\ ${ }^{5}$ Department of Physics, Philipps-Universität Marburg, D-35032, Marburg, Germany \\ ${ }^{6}$ Donostia International Physics Center (DIPC), 20018, San Sebastián, Spain \\ ${ }^{7}$ Departamento de Física de Materiales, Facultat de Ciencias Químicas, UPV/EHU Apdo. 1072, 20080, Donostia/ San Sebastián, Spain
}

(Received 6 June 2016; revised manuscript received 29 September 2016; published 31 October 2016)

\begin{abstract}
The geometrical and electronic properties of the monolayer (ML) of tetracene (Tc) molecules on $\operatorname{Ag}(111)$ are systematically investigated by means of DFT calculations with the use of a localized basis set. The bridge and hollow adsorption positions of the molecule in the commensurate $\gamma-\mathrm{Tc} / \mathrm{Ag}(111)$ are revealed to be the most stable and equally favorable irrespective to the approximation chosen for the exchange-correlation functional. The binding energy is entirely determined by the long-range dispersive interaction. The former lowest unoccupied molecular orbital remains being unoccupied in the case of $\gamma-\operatorname{Tc} / \operatorname{Ag}(111)$ as well as in the $\alpha$ phase with increased coverage. The unit cell of the $\alpha$ phase with point-on-line registry was adapted for calculations based on the available experimental data and computed structures of the $\gamma$ phase. The calculated position of the $\operatorname{Tc} / \operatorname{Ag}(111)$ interface state is found to be noticeably dependent on the lattice constant of the substrate, however its energy shift with respect to the Shockley surface state of the unperturbed clean side of the slab is sensitive only to the adsorption distance and in good agreement with the experimentally measured energy shift.
\end{abstract}

DOI: 10.1103/PhysRevB.94.155452

\section{INTRODUCTION}

Organic molecular thin films are currently of great interest because of their possible applications in micro- and optoelectronic devices $[1,2]$. Their properties depend on the nature of the interface between the molecular layer and the substrate $[3,4]$. The performance of the molecular devices is considerably conditioned by the efficiency of charge transfer across the interface, which in turn is governed by the relative alignment of molecular energy levels with respect to the Fermi level of the metal substrate as well as the overlap between molecular and substrate wave functions [5,6]. The presence of interface electronic states (ISs) [7-9] is an additional agent influencing the overall charge transfer, albeit the role of these states in the process and mechanism of their formation are not yet fully understood [10]. On the one hand, such hybrid states can be formed as the result of the chemical interaction of molecular orbitals with metallic states [7,11-13]. On the other hand, in many cases the symmetry breaking at the metal/organic interface alone can lead to new interface electronic states, analogs to the Shockley state of clean metal surfaces [8,14-17]. Theoretical investigations of these types of organic/metal interface states (IS) have focused on molecules with a perylene core and carboxylic end groups, i.e., NTCDA and PTCDA $[9,14,15,18,19]$. The calculations revealed that the IS has its maximum probability density between the topmost metallic layer and the plane of carbon atoms. The IS wave function shows the penetration into the metal substrate similar to the Shockley surface state (SS) [20]. At the same time, the lateral corrugation of the IS local density of states above the

\footnotetext{
*nza@yandex.ru
}

metal substrate resembles that of organic molecular orbitals [9,14-16,18,19].

The interaction of NTCDA and PTCDA with many metal substrates, however, is not of purely van der Waals type. On $\operatorname{Ag}(111)$ and $\operatorname{Ag}(100)$ a lowering and partial filling of the lowest unoccupied molecular orbital of the molecules is observed [21]. In such a situation, it is difficult to assess to what extent this type of chemical hybridization influences the interface state and whether it attains similar properties for the case of physisorption. Here we thus consider a model system with a weaker molecule-substrate interaction, tetracene/ $\operatorname{Ag}(111)$

Tetracene $(\mathrm{Tc})$ is an organic molecule with planar aromatic structure $\left(\mathrm{C}_{18} \mathrm{H}_{12}\right)$ and it is one of the most promising organic semiconductors for practical applications due to its high charge carrier mobility [22,23]. A number of arrangement patterns (or ordered phases) of Tc molecules on the $\operatorname{Ag}(111)$ surface has been observed as a function of coverage [24,25]. The compressed monolayer (ML) $\alpha$ phase with point-on-line type of commensurability, which can undergo spontaneous structural transformation[25], has been studied in detail by different experimental techniques [26,27]. Additionally, the fully commensurate $\gamma$ phase with submonolayer coverage has been discovered rather recently [24]. Both phases are characterized by the same orientation of the molecule with respect to the substrate, and they are suitable for a computational study of the effect of coverage on the adsorption geometry and electronic properties. This work is intended to get a better understanding about the interaction between Tc molecules and the silver (111) surface, as well as its effect on the energy levels alignment and the interface electronic states.

In this study we have examined $\gamma$ - and $\alpha$-Tc/Ag(111) by means of density functional theory (DFT) calculations 
with numerical pseudoatomic orbitals. In the case of largesize systems, the localized basis functions offer obvious advantages over the plane waves, especially for the reasonable description of interface states, which requires metal surfaces to be represented by a quite thick slab [19]. However, an accurate description of delocalized surface states with the use of pseudoatomic orbitals needs careful handling [28]. Here we explore a few ways of representation of substrate wave functions by localized numerical basis functions and show that the most proper way is to use different cutoff radii of these basis functions for bulk and for surface Ag atoms. We reveal that the energy of both the surface state and the interface state depends directly on the substrate lattice constant. The binding between $\mathrm{Tc}$ and $\operatorname{Ag}(111)$ turns to be described correctly if one takes into account the long-range dispersive forces that fully determines the substrate-adsorbate interaction in the interface under study. Our results are found to provide overall good description of the available experimental data on Tc/Ag(111).

\section{METHODS}

The first-principles electronic structure calculations are performed within the DFT as implemented in the SIESTA code [29,30]. Localized pseudoatomic orbitals were used for the wave function representation, and deep core potentials were represented by norm-conserving Troullier-Martins pseudopotentials [31] in the Kleynman-Bylander [32] nonlocal form. The conventional generalized gradient approximation (GGA) was chosen for the exchange-correlation functional with the PBE [33] parametrization. Long-range dispersion forces were introduced by using the optB88-vdW functional of the vdW-DF2 approach especially adapted for solids [34,35].

The double- $\zeta$ polarized (DZP) basis set with the energy shift of $10 \mathrm{meV}$ and generated by a soft confinement scheme was used for hydrogen (with the cutoff radius $r_{c}$ of 7.2 a.u.), carbon (5.95 a.u.), and silver (9.73 a.u.) atoms. Such basis functions for silver provide the enlarged equilibrium lattice constant $a=4.23 \AA$ for both the GGA-PBE and the optB88$\mathrm{vdW}$ functional. The indicated cutoff-radius value for silver was taken to reach the convergence of the lattice constant. However, the reasonable silver bulk properties were found with the shorter radius $r_{c}=7.03$ a.u. of the basis orbitals. Actually, the equilibrium lattice constant $a=4.16 \AA$ and the bulk modulus $B=114 \mathrm{GPa}$ were obtained with the GGA-PBE functional, and similar values $(a=4.17 \AA, B=119 \mathrm{GPa})$ with the optB88-vdW one.

On the one hand, for a good description of the silver (111) surface, i.e., to obtain accurate values of the surface energy, work function, energy of the surface state, and its decay into the vacuum, one needs to use the basis functions with a quite large $r_{c}$ [28]. It was also shown [36] that the long-range orbitals are needed to minimize the basis set superposition error (BSSE) and to reproduce the binding energy of plane-wave calculations. On the other hand, a shorter $r_{c}$ provides the bulk properties consistent with the experiment, which are important to be described properly, because an increase of the lattice constant causes the energy upshift of the surface-state position of the $\mathrm{Ag}(111)$ surface. Moreover, as calculations show, the adsorption distance of aromatic molecules on metallic surfaces depends on the parameter $r_{c}$ of the substrate: the shorter the radius we chose the smaller the distance we have [37]. Thereby, in examining the geometrical and electronic properties of the metal-organic interface under study, we consider three models to the silver-substrate description.

In the first model, the experimental value for the silver lattice constant $(a=4.09 \AA)$ and the largest radius of the basis functions ( $r_{c}=9.73$ a.u.) are used. All silver atoms in the slab are pinned to their bulk positions, but the atomic positions of the molecular monolayer are allowed to be relaxed. In the second model, two types of basis functions are used for silver atoms: $r_{c}=9.73$ a.u. for atoms in the upper- and lowermost silver layers of the slab (the external atoms) and $r_{c}=7.03$ a.u. for the rest of atoms (the internal atoms). As was shown in Ref. [28], such an approach improves the description of clean surfaces. The lattice constant is set to its equilibrium value as found with the basis functions of the internal atoms. Along with atoms in the molecular ML, the positions of the atoms in the two uppermost silver layers are optimized as well. We improve thus the description of surface electronic bands and substrate bulk properties simultaneously. It is worth noting that the use of orbitals with the largest $r_{c}$ for all atoms in the slab leads to insignificant changes of the electronic band structure, but vastly increases the computational cost. In order to demonstrate it, inthe thirdtype of substrate handling all silver atoms are taken with the large $r_{c}=9.73$ a.u. In this case, the equilibrium lattice constant has the value of $a=4.23 \AA$. Again, only the positions of the silver atoms in the two uppermost layers of the slab are allowed to be optimized. Hereafter, we refer to these models of the substrate description as to models 1,2 , and 3 , respectively.

The scheme of periodically repeated slabs are used to describe the infinite close packed face-centered cubic $\operatorname{Ag}(111)$ surface. Interaction between the periodic images of the systems in the direction perpendicular to the surface (in the $z$ direction) is suppressed by the large size of the cell along this direction, imposing a vacuum layer of about $11 \AA$. A uniform mesh for numerical integration and solution of the Poisson equation is specified by the energy cutoff of $250 \mathrm{Ry}$. The substrate was represented by four layers during structural relaxation. To analyze the bands alignment, we calculated the band structure of $\gamma$ - and $\alpha$-Tc/Ag(111) with the use of a 12-layer silver slab to make the study feasible. The convergence of the IS energy measured from the Fermi level with respect to the slab thickness was examined for $\gamma$-Tc/Ag(111) within model 2 by considering additionally the slab of 16, 19, 22, and 25 layers. The molecular monolayer is applied to one side of the substrate only. We use the k-point sampling of the surface Brillouin zone based on the Monkhorst-Pack scheme with $22 \mathbf{k}$ points. The iterative modified Broyden procedure [38] is applied to reach stable structures. All the considered geometries are relaxed until interatomic forces were smaller than $0.02 \mathrm{eV} / \AA$.

The spatial distribution of the interface state was computed with the help of the OpenMX (version 3.7) DFT code [39-42]. For silver atoms, two types of basis functions with different cutoff radii of 7.0 and 9.0 bohrs (as in model 2), but with the same size $s 2 p 2 d 2 f 1$ were used, while for hydrogen and carbon atoms we chose H7.0-s $2 p 1$ and $C 7.0-s 2 p 2 d 1$, respectively. The notation in the last case means that two primitive orbitals for each $s$ and $p$ states and one primitive orbital for the $d$ states with the cutoff radius of 7.0 bohrs 
were used. The real-space grid for numerical integration was specified by the energy cutoff of $250 \mathrm{Ry}$. The total-energy convergence was better than $0.027 \mathrm{meV}$. The surface Brillouin zone (SBZ) of the supercell was sampled with a $6 \times 6 \times 1$ mesh of $\mathbf{k}$ points. The calculation were performed within GGA-PBE approximation.

The alignment of the Tc molecules on the silver (111) surface in the $\gamma$ phase and its surface unit cell were determined in accordance with the experimental data [24]. The unit cell characterized by the structural matrix $\left(\begin{array}{cc}3 & 2 \\ -5 & 2\end{array}\right)$ contains only one molecule with the coverage of $83 \%$, being commensurate with the hexagonal lattice of $\operatorname{Ag}(111)$. The orientation of the molecular longitudinal axis coincides with [01]ㅣ directions of the silver substrate. To find the most favorable structure, four different adsorption sites were used as the starting location of the Tc-molecule center (see Fig. 1) for structure optimization. The on-top $\Gamma_{0}$ position of Tc was proposed in the experiment as the most probable one [24]. Also, the bridge $\left(\Gamma_{2}\right)$, hollow $\left(\Gamma_{3}\right)$, and long bridge $\left(\Gamma_{1}\right)$ adsorption sites were considered (Fig. 1).

The unit cell of the $\alpha$ phase contains one Tc molecule with the coverage of $100 \%$, which has the point-on-line coincidence with the substrate and is described by the noninteger matrix $\left(\begin{array}{ll}3.1 & 2 \\ -2.25 & 3\end{array}\right)[24,26]$. The commensurate unit cell can be obtained by a tenfold increase of the first unit vector and fourfold of the second one. As a result, the unit cell contains already 40 molecules with their own adsorption sites. By neglecting the shifts of the adsorption site along the first unit vector (it is only 0.1 of the silver interatomic distance), the unit cell can be tenfold shortened in this direction. Such a reduced unit cell contains only four molecules on different adsorption sites and has the $101 \%$ coverage. This adapted unit cell is used in our calculations of the electronic structure of the $\alpha$ -

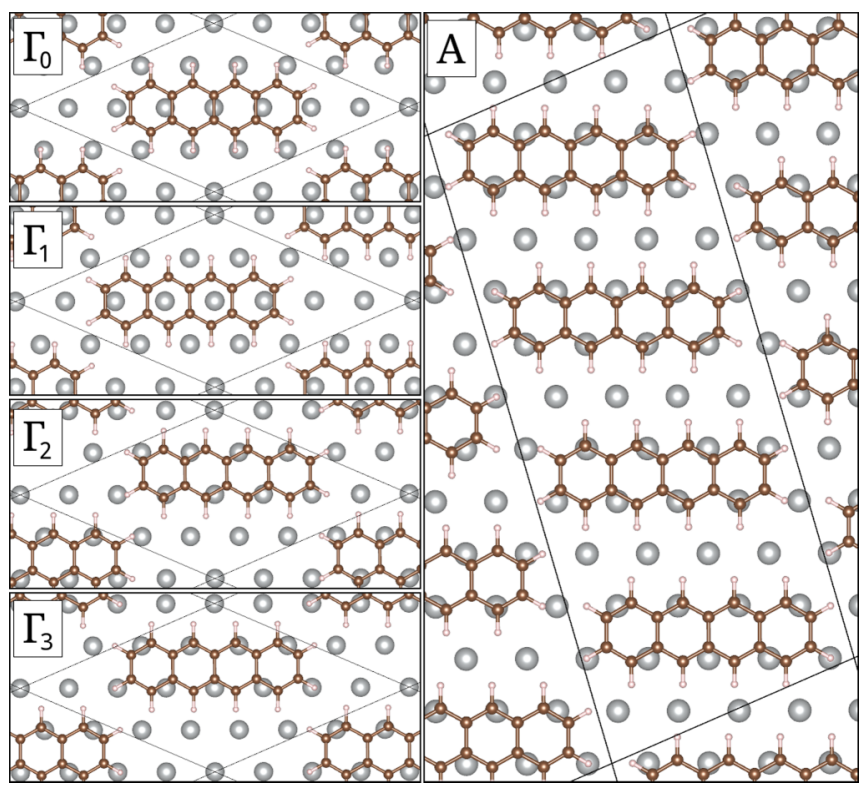

FIG. 1. Different adsorption sites of $\gamma-\mathrm{Tc} / \mathrm{Ag}(111) . \Gamma_{0}$ is the top site, $\Gamma_{1}$ is the long bridge site, $\Gamma_{2}$ is the bridge site, and $\Gamma_{3}$ is the hollow site. The commensurate unit cell with four Tc molecules represents the monolayer of $\alpha$-Tc/Ag(111).
Tc/Ag(111) interface, which are performed within model 2 only (see Fig. 1).

The binding energy is introduced in the conventional way as the difference between the total energy of the whole system $E_{\text {sys }}$ and the sum of the energies of independently optimized freestanding monolayer $E_{\mathrm{ml}}$ and silver substrate $E_{\text {sub. The }}$ counterpoise correction [43] is exploited to estimate the basis set superposition error. To this end, the binding energy is written as follows:

$$
E_{b}^{(\mathrm{cp})}=E_{\mathrm{int}}^{(\mathrm{cp})}-E_{\mathrm{def}}^{\mathrm{ml}}-E_{\mathrm{def}}^{\mathrm{sub}} .
$$

Here the substrate-adsorbate interaction energy is expressed as $E_{\mathrm{int}}^{(\mathrm{cp})}=E_{\mathrm{sys}}-E_{\mathrm{ml}}^{*(\mathrm{cp})}-E_{\mathrm{sub}}^{*(\mathrm{cp})}$, where both constituents of the system can be counterpoise corrected (as indicated by the superscript in parentheses) or not, and they both are cut from optimized geometry of the whole system. The deformation term is simply the difference between the energy of the optimized freestanding monolayer (or substrate) $E_{\mathrm{ml}(\text { sub) }}$ and the energy of the monolayer (substrate) $E_{\mathrm{ml}(\mathrm{sub})}^{*}$ clamped in the adsorption geometry.

\section{RESULTS}

The calculated binding energy of $\gamma$-Tc/Ag(111) listed in Table I evidences that Tc molecules prefer to be lined up directly above the silver rows by their external carbon atoms, i.e., by those linked with hydrogen (Fig. 1). The long bridge $\left(\Gamma_{1}\right)$ and on-top adsorption geometries $\left(\Gamma_{0}\right)$ provide disadvantageous alignments of Tc molecules on $\operatorname{Ag}(111)$; in both cases carbon atoms are located upon the interstitial sites of the underlying silver layer (Fig. 1). The same trends in adsorption geometry were revealed in the recent calculations of the $\mathrm{Tc} / \operatorname{Ag}(110)$ interface [44]. Because of the obvious disadvantage of the $\Gamma_{1}$ geometry, it will be excluded from our further analysis, but despite the unfavorable adsorption structure of $\Gamma_{0}$, it will be considered as the reference structure proposed by experimentalists [24]. The bridge $\left(\Gamma_{2}\right)$ and hollow $\left(\Gamma_{3}\right)$ sites are the most favorable adsorption sites of $\gamma$-Tc and they both provide similar binding energies (Table I). The above statement holds true irrespectively of the exchange-correlation approximation and type of the substrate model used.

\section{A. Adsorption geometry}

For the same adsorption site, the vertical separation of carbon atoms as found with the GGA-PBE functional within models 1 and 2 is characterized by the fairly close averaged values (see Table I); the difference is merely of $0.03 \AA$ in the case of the on-top and bridge positions of the molecule. Model 3 gives the noticeably smaller adsorption height for all adsorption sites. It is worth also noting that the Tc molecule is rather distorted on the $\operatorname{Ag}(111)$ surface. Actually, the $z$ coordinate of the molecule atoms varies within the range of $0.07-0.10 \AA$. It is clearly seen in Table I,where the superscript in parentheses denotes the maximal difference between the perfect $z$ coordinate of the topmost silver layer and the $z$ position of carbon and hydrogen atoms of the molecule. For the on-top adsorption geometry, the distortion is found along the longitudinal axis only, and the center of the molecule is farther from the substrate than the molecule edges. The attractive 
TABLE I. Vertical adsorption distances (in $\AA$ ) and binding energies (in eV) of $\gamma$-Tc/Ag(111) for different models of the substrate. The distance is represented by the average value of the normal distribution of vertical separations for carbon and hydrogen atoms. The lattice constant of the silver substrate (in $\AA$ ) for every model is also given in square brackets. The superscript in parentheses denotes the spread of the vertical separations for different $\mathrm{C}$ or $\mathrm{H}$ atoms in the ML, reflecting the distortion of the Tc molecule deposited on top of the Ag(111) surface.

\begin{tabular}{|c|c|c|c|c|c|c|c|c|c|}
\hline & $d_{z}(\mathrm{C})$ & $\begin{array}{r}d_{z}(\mathrm{H}) \\
\operatorname{Top}\left(\Gamma_{0}\right)\end{array}$ & $E_{b}$ & $d_{z}(\mathrm{C})$ & $\begin{array}{c}d_{z}(\mathrm{H}) \\
\text { Bridge }\left(\Gamma_{2}\right)\end{array}$ & $E_{b}$ & $d_{z}(\mathrm{C})$ & $\begin{array}{c}d_{z}(\mathrm{H}) \\
\text { Hollow }\left(\Gamma_{3}\right)\end{array}$ & $E_{b}$ \\
\hline \multicolumn{10}{|l|}{ GGA-PBE } \\
\hline Model 1 [4.09] & $3.60^{(0.07)}$ & $3.57^{(0.09)}$ & -0.40 & $3.52^{(0.09)}$ & $3.48^{(0.10)}$ & -0.42 & $3.51^{(0.14)}$ & $3.45^{(0.23)}$ & -0.42 \\
\hline Model 2 [4.16] & $3.57^{(0.09)}$ & $3.52^{(0.10)}$ & -0.48 & $3.49^{(0.15)}$ & $3.44^{(0.20)}$ & -0.50 & $3.41^{(0.15)}$ & $3.37^{(0.21)}$ & -0.49 \\
\hline Model 3 [4.23] & $3.41^{(0.02)}$ & $3.38^{(0.03)}$ & -0.32 & $3.32^{(0.08)}$ & $3.27^{(0.13)}$ & -0.35 & $3.32^{(0.11)}$ & $3.27^{(0.18)}$ & -0.35 \\
\hline \multicolumn{10}{|l|}{ optB88-vdW } \\
\hline Model 1 [4.09] & $3.22^{(0.02)}$ & $3.19^{(0.01)}$ & -1.96 & $3.14^{(0.04)}$ & $3.11^{(0.06)}$ & -2.05 & $3.16^{(0.08)}$ & $3.13^{(0.17)}$ & -2.05 \\
\hline Model 2 [4.17] & $3.21^{(0.03)}$ & $3.19^{(0.03)}$ & -1.97 & $3.16^{(0.07)}$ & $3.13^{(0.12)}$ & -2.06 & $3.15^{(0.10)}$ & $3.14^{(0.18)}$ & -2.06 \\
\hline Model 3 [4.23] & $3.10^{(0.04)}$ & $3.09^{(0.04)}$ & -1.86 & $3.00^{(0.05)}$ & $2.98^{(0.08)}$ & -1.97 & $3.02^{(0.06)}$ & $3.01^{(0.11)}$ & -1.97 \\
\hline
\end{tabular}

interaction between carbon and underlying silver atoms results in more complicated distortion of Tc, when the center of the latter occupies the bridge or hollow site. For instance, the lateral slop of Tc in the hollow $\left(\Gamma_{3}\right)$ site takes place, i.e., the side of the molecule, on which the external carbon atoms are located directly over the silver atoms is about $0.08 \AA$ closer to the substrate than the side with carbon atoms located over the interstitials (see Fig. 1).

The inclusion of the long-range interaction within the optB88-vdW approximation gives rise to a reduction of the vertical adsorption distance (Table I). Models 1 and 2 provide almost identical vertical separations and binding energies. As it was before, model 3 (the enlarged Ag lattice constant) results in shorter separations and a weaker binding. The adsorption geometry $\Gamma_{0}$ is characterized by the nearly flat shape of Tc, while, as in the previous case, the molecule is more distorted in $\Gamma_{2}$ and $\Gamma_{3}$ in accordance with the mutual alignment of carbon and silver atoms. Moreover, in the case of the hollow adsorption site the slop of the Tc molecule has the same value regardless the approximation chosen for the exchange-correlation functional.

The experimental estimation of the binding energy for $\alpha$-Tc by the thermal desorption study [27] is about $1 \mathrm{eV}$ (1.4 $\mathrm{eV}$ in the zero-coverage limit), while for $\gamma$-Tc it is expected to be a slightly higher, because of the smaller coverage. The calculated binding energy of the $\Gamma_{2}$ structure is thus considerably underestimated within the GGA-PBE calculations (Table I); as compared with the experimental value the energy of $0.5 \mathrm{eV}$ is half as much. On the contrary, the calculation with the optB88-vdW functional provides $E_{b}$, which is twice as much in comparison with the experiment (Table I).

The detailed insight into the substrate-adsorbate interaction is done for the bridge $\left(\Gamma_{2}\right)$ geometry, because it is one of the most energetically favorable structures. The major part of the binding energy is the interaction energy (Table II), while the deformation energy of both the molecular monolayer and the topmost layers of the substrate is of $\sim 20 \mathrm{meV}$ in the PBE calculations and slightly larger in the case of the optB88-vdW functional. As compared with the GGA-PBE values, the longrange dispersive interaction gives rise to a fourfold increase in the interaction energy.

The binding energy found with the use of localized basis functions tends to be overestimated by reason of BSSE, which should be corrected. There is a noticeable reduction in counterpoise corrected substrate-adsorbate interaction, because of quite a large area of the Tc molecule for both functionals used. In the GGA-PBE calculations, the considered $\Gamma_{2}$ structure

TABLE II. Energy terms (eV) of Eq. (1) (with and without the BSSE correction), the energies of the former frontier orbitals of the Tc molecules $(\mathrm{eV})$ as measured from the Fermi energy of the interface, and the amount of charge $(-e)$ in the molecular monolayer region for the bridge adsorption geometry $\Gamma_{2}$ of the $\gamma$ and $\alpha$-Tc/Ag(111) interfaces.

\begin{tabular}{|c|c|c|c|c|c|c|}
\hline & $d_{z}$ & $E_{\mathrm{int}} / E_{\mathrm{int}}^{\mathrm{cp}}$ & $E_{\mathrm{def}}^{\mathrm{ml}}$ & $E_{\mathrm{def}}^{\mathrm{sub}}$ & $E_{b} / E_{b}^{\mathrm{cp}}$ & $Q\left(z_{0}\right) / Q^{\mathrm{cp}}\left(z_{0}\right)$ \\
\hline & $\gamma-\mathrm{Tc}$ & & & & & \\
\hline \multicolumn{7}{|l|}{ GGA-PBE } \\
\hline Model 1 [4.09] & 3.52 & $-0.43 /-0.02$ & 0.00 & & $-0.42 /-0.01$ & $0.10 / 0.14$ \\
\hline Model 2 [4.16] & 3.49 & $-0.53 /-0.02$ & -0.01 & -0.01 & $-0.50 / 0.00$ & $0.07 / 0.12$ \\
\hline $\begin{array}{l}\text { Model } 3 \text { [4.23] } \\
\text { optB88-vdW }\end{array}$ & 3.32 & $-0.37 /-0.01$ & -0.01 & -0.02 & $-0.35 / 0.01$ & $0.12 / 0.16$ \\
\hline Model 1 [4.09] & 3.14 & $-2.06 /-1.50$ & -0.01 & & $-2.05 /-1.49$ & $0.13 / 0.20$ \\
\hline Model 2 [4.17] & 3.16 & $-2.09 /-1.51$ & -0.01 & -0.01 & $-2.06 /-1.48$ & $0.09 / 0.18$ \\
\hline Model 3 [4.23] & $\begin{array}{l}3.00 \\
\alpha-\mathrm{Tc}\end{array}$ & $-2.01 /-1.54$ & -0.01 & -0.03 & $-1.97 /-1.50$ & $0.14 / 0.21$ \\
\hline \multicolumn{7}{|l|}{ optB88-vdW } \\
\hline Model 2 [4.17] & 3.16 & $-1.87 /-1.44$ & -0.01 & -0.02 & $-1.84 /-1.41$ & $0.10 / 0.16$ \\
\hline
\end{tabular}


becomes even unstable (Table II), being characterized by the positive binding energy. In the case of the optB88-vdW functional, the counterpoise corrected binding energy comes closer to the experimental value [27], but it is still overestimated. Thus, the binding of the molecular monolayer with silver in $\gamma$-Tc/Ag(111) is fully determined by the long-range dispersive forces. Note also that the BSSE-free values of the binding energy are almost the same for all models of the substrate; its deviation is about $20 \mathrm{meV}$.

The calculation of $\alpha-\mathrm{Tc} / \mathrm{Ag}(111)$ was performed in model 2 with the optB88-vdW functional only. As was described above, the coverage in the adapted unit cell coincides well with the experimental one. The adsorption sites for Tc were chosen in accordance with the $\gamma$-phase calculations, i.e., the external carbon atoms reside above the silver atoms (Fig. 1) as in the bridge and hollow adsorption sites. The averaged vertical separations for carbon (3.16 $\AA$ ) and hydrogen $(3.13 \AA$ ) atoms are very close to the $\gamma$-phase results (see Table II), but the binding energy becomes smaller as compared with the $\gamma$ phase because of the increased coverage.

\section{B. Charge transfer and bands alignment}

Due to the substrate-adsorbate interaction, the real space electron-density rearrangement occurs in the interface region. The charge transfer between the substrate and the molecular monolayer can be extracted from the laterally averaged electron-density difference, which is calculated as

$$
\Delta n(z)=n_{\text {sys }}(z)-n_{\mathrm{ml}}(z)-n_{\text {sub }}(z) .
$$

Here $n_{\text {sys }}$ is the charge density of the interface under study averaged over the $x y$ plane within the unit cell, and $n_{\mathrm{ml}}$ and $n_{\text {sub }}$ are the charge densities of the monolayer and the substrate, respectively. A positive value of $\Delta n(z)$ indicates the gain in electron density upon adsorption, while its negative value means the loss of electrons. As clearly seen in Fig. 2, the regions around molecular plane lose electrons. The amount of change transfer can be thus determined by the function $Q(z)=\int_{z_{\mathrm{vac}}}^{z} \Delta n\left(z^{\prime}\right) d z^{\prime}$, with $z_{\mathrm{vac}}$ being the point in the vacuum region, where $\Delta n(z)$ is zero. Since the integration is started from the clean side of the slab, which is on the left in Fig. 2, the positive values of $Q(z)$ at a given $z$ provide the amount of charge, which flow from the right side into the left side with respect to this $z$ and vice versa for negative values. If we fix the boundary between the substrate and the adsorbate at the point in the interface region $z_{0}$, where $\Delta n(z)$ crosses zero right on the left of its global minimum, then the positive $Q\left(z_{0}\right)$ will determine the charge transfer from molecule to metal. The $Q\left(z_{0}\right)$ values listed in Table II are quite close to each other, depending, nevertheless, on the adsorption distance. Actually, it tends to be increased with shortening of the molecule-substrate separation. Additionally, $\Delta n$ is sensitive to the overlap between molecular and substrate wave functions, as it is clearly seen on example of model 2 [see Figs. 2(b) and 2(d)], where for the topmost silver atoms the basis functions with increased $r_{c}$ were used. The effect of the wave functions overlap on the amount of charge transfer is also observed in the case of the counterpoise corrected charge difference. The superposition of substrate and molecule basis functions is expected to cause an error in the charge density similar to
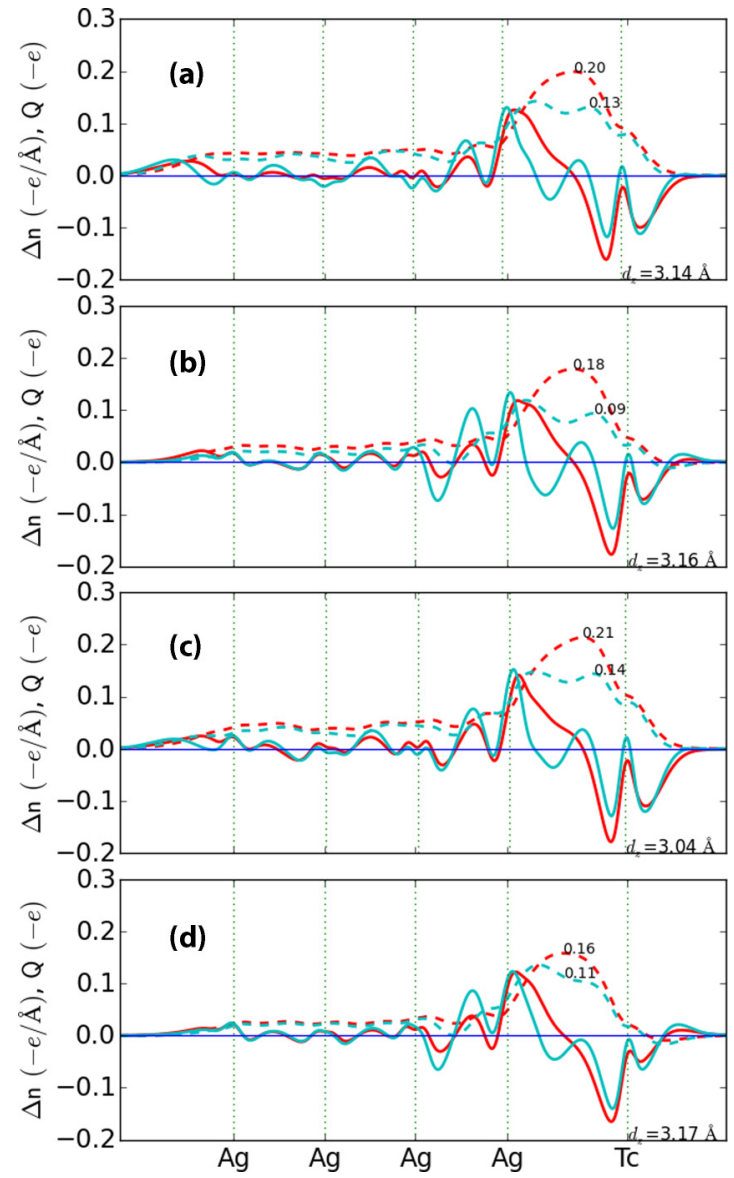

FIG. 2. The charge density difference $\Delta n(z)$ averaged in $x y$ plane (solid lines) and the respective amount of charge transfer $Q(z)$ (dashed lines) with (red) and without (cyan) the BSSE correction for the bridge $\left(\Gamma_{2}\right)$ adsorption site. (a) Model 1, (b) model 2, (c) model 3 of $\gamma$-Tc, and (d) model 2 of $\alpha$-Tc.

the interaction energy. The amount of charge transfer into the substrate is noticeably increased due to the redistribution of the BSSE free charge density (see Table II), and the accumulation of charge density upon silver layer becomes evident (see Fig. 2).

The aforementioned behavior of $\Delta n(z)$ and $Q(z)$ can be interpreted in terms of the push-back effect [45], which is typical for organic molecules physisorbed onto metallic surfaces. Actually, the repulsive exchange interaction between the molecular and substrate electrons, i.e., Pauli repulsion, leads to the molecular electronic cloud pushes the substrate electronic cloud back into the metal. The same mechanism is responsible for charge transfer in the repeatedly described PTCDA/Au(111) interface [46,47].

The charge density difference for the $\alpha$ phase with increased coverage of $\mathrm{Tc}$ on $\mathrm{Ag}(111)$ provides almost the same outflow of charge from the ML as in the $\gamma$ phase, i.e., the increase of the charge density in the ML has a minimal effect on the amount of charge transfer. Note, a negative change in the work function upon adsorption of Tc has been experimentally observed [48], suggesting donation of negative charge from the molecule to the Ag substrate, which was roughly estimated 


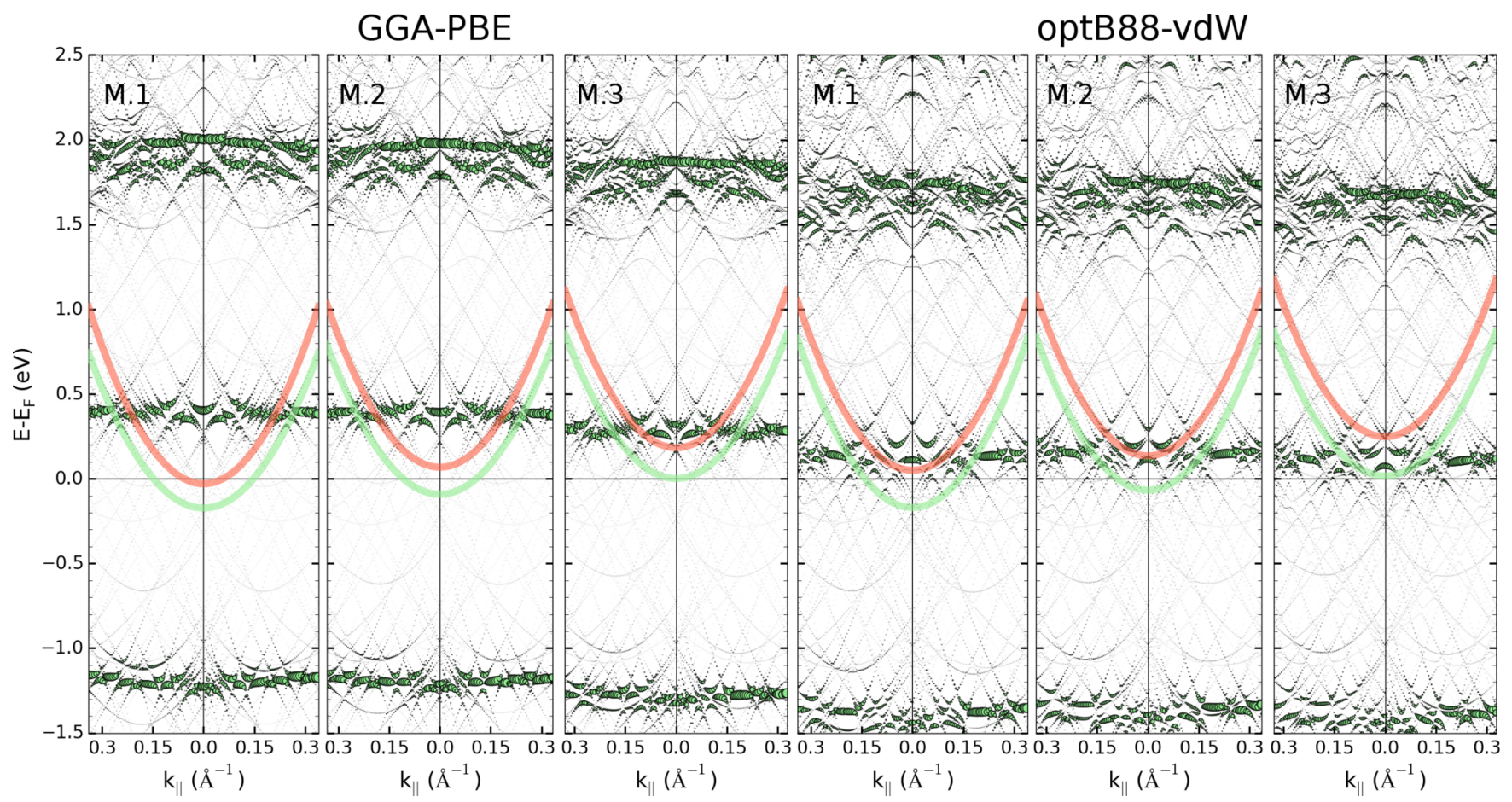

FIG. 3. Band structure of the $\gamma-\mathrm{Tc} / \mathrm{Ag}(111)$ interface projected onto the $p_{z}$ molecular states. The dispersive interface (red thick line) and surface (green) states are presented as well.

afterwards [27]. The present calculation is thus consistent with these experimental findings.

The fact that the molecular ML loses electrons implies that there is an electron donation from Tc to the substrate involving many formerly occupied molecular orbitals, and that the LUMO (the lowest unoccupied molecular orbital) of Tc is still empty after the interaction with silver. The projected band structure onto $p_{z}$ states of carbon atoms in the directions of the reciprocal lattice vectors was calculated for the system where the silver substrate is represented by a 12-layer slab (Fig. 3). As clearly seen in the figure, the energy-bands alignment of the interacting systems with respect to the Fermi level ensures that the LUMO of Tc remains unoccupied and nondispersive, residing close to the Fermi level, especially in the optB88-vdW calculations.

The band structure calculated within models 1 and 2 shows similar energy values for the unoccupied LUMO and LUMO+1 (Table III), while these orbitals are shifted down in the case of model 3 for both functionals (see Table II). This energy downshift can be explained by the decrease of the substrate-adsorbate distance. Therefore, the calculated energy of the LUMO with the GGA-PBE functional $(390 \mathrm{meV})$ is higher than the value of $150 \mathrm{meV}$ obtained with the optB88$\mathrm{vdW}$ one (Table III). Since the adsorption height of the $\alpha$ phase considered in model 2 within optB88-vdW approximation is the same as that of the $\gamma$ phase, the energy of the LUMO has a close value too, but now this band is characterized by a larger width. Note that the calculated LUMO energies do not coincide with experimental values due to the well-known limitation of the DFT in describing quasiparticle states [49-51].

The adsorption of the Tc monolayer on $\operatorname{Ag}(111)$ in the $\gamma$ phase results in an upshift of the silver surface state (SS). The same transformation of the SS manifests itself after the adsorption of NTCDA and PTCDA $[9,14,19]$ or phthalocyanine [16] molecules on the same substrate, whereas the downshift of the SS was observed for pentacene molecules absorbed on $\mathrm{Cu}(110)$ [52]. The band structure projected onto the $p_{z}$ states of silver atoms located in the uppermost substrate layer covered by the $\mathrm{ML}$ or the clean lowermost silver layer directly reveals the IS or the clean-side SS of the slab, respectively. Both states are shown in Fig. 3 by red (IS) and green (SS) dispersive curves.

The energies of both states at the $\bar{\Gamma}$ point depend strongly on the type of the substrate model. Namely, they are very sensitive to the silver lattice constant; the greater the equilibrium lattice constant that the bulk silver possesses the higher the SS energy we obtain. The same behavior of the SS is observed for the clean $\operatorname{Ag}(111)$ surface. Nevertheless, as follows from Table III the energy difference $\delta E$ of the IS and SS keeps close value in models 1 and 2, and it has a larger value in model 3 , because of

TABLE III. The energies (in meV) of the interface state $\left(E_{\mathrm{IS}}\right)$ and the surface state $\left(E_{\mathrm{SS}}\right)$ and their difference $\delta E$ for the bridge $\left(\Gamma_{2}\right)$ adsorption position. $\mathrm{Tc} / \mathrm{Ag}(111)$ silver slab has the 12-layer thickness.

\begin{tabular}{lccccccccc}
\hline \hline & $\begin{array}{c}E_{\mathrm{IS}} \\
\text { GGA-PBE }\end{array}$ & \begin{tabular}{c}
$E_{\mathrm{SS}}$ \\
\multicolumn{2}{c}{$\delta E$}
\end{tabular} & $E_{\mathrm{LUMO}}$ & $\begin{array}{c}E_{\mathrm{IS}} \\
\text { optB88-vdW }\end{array}$ & \begin{tabular}{c}
$E_{\mathrm{SS}}$ \\
\multicolumn{1}{c}{$\delta E^{2}$}
\end{tabular} & $E_{\mathrm{LUMO}}$ \\
\hline $\boldsymbol{\gamma}$-Tc & & & & & & & & \\
Model 1 & -20 & -172 & 152 & 390 & 50 & -164 & 214 & 140 \\
Model 2 & 68 & -92 & 160 & 390 & 135 & -70 & 205 & 150 \\
Model 3 & 183 & 1 & 182 & 280 & 248 & 16 & 232 & 110 \\
$\alpha$-Tc & & & & & & & & \\
Model 2 & & & & & 175 & -60 & 235 & 150 \\
Expt. [24] & & & & & & 220 & 830 \\
\hline \hline
\end{tabular}


TABLE IV. Dependence of the IS (SS) energy and the related difference $\delta E$ (in $\mathrm{meV}$ ) with respect to the number of the substrate layers. The bridge $\left(\Gamma_{2}\right)$ adsorption geometry within model 2 with the optB88-vdW functional is considered.

\begin{tabular}{lccc}
\hline \hline & $E_{\mathrm{IS}}$ & $E_{\mathrm{SS}}$ & $\delta E$ \\
\hline 12 & 135 & -70 & 205 \\
16 & 125 & -30 & 155 \\
19 & 100 & -30 & 130 \\
22 & 100 & -20 & 120 \\
25 & 100 & -20 & 120 \\
\hline \hline
\end{tabular}

the smaller adsorption distance [9,19]. Definitely, the GGA$\mathrm{PBE}$ calculations provide a smaller $\delta E$ as compared with that found with the optB88-vdW functional for the same reason.

\section{Interface state}

Now we turn to the analysis of the convergence of the IS energy with respect to the slab thickness. The aforementioned dependence of $\delta E$ on the substrate model is irrespective to the slab thickness, while within a given model the IS (SS) energy measured from the Fermi level and, hence $\delta E$, can be rather sensitive to the number of Ag-atomic layers in the slab modeling the substrate. To find a converged IS energy for the Tc/Ag(111) interfaces, we will focus on optB88-vdW calculations within model 2 only.

The performed careful analysis of variations of the IS and SS energy with the number of substrate layers shows that the well converged energies (100 and $-20 \mathrm{meV}$, respectively) can be reached for the slab containing $22 \mathrm{Ag}$ layers (see Table IV). In contrast to the chemisorbed monolayers of PTCDA and NTCDA on Ag(111), where the 12-layer slab ensures the convergence of the IS energy [19], the physisorbed Tc monolayer demands markedly more silver layers. The substrate-adsorbate interaction accelerates convergence of the IS and clean side SS with respect to the slab thickness and facilitates their decoupling. As clearly seen in Fig. 4(a), the IS and SS are decoupled quite well for the slab containing 19 layers. The IS wave function is almost completely localized on the side with the adsorbate, whereas the SS resides on the opposite clean side of the slab. The strong chemical interaction between the functional groups and metallic atoms in the case of NTCDA (PTCDA) MLs on Ag(111) [19], results in a shorter adsorption distance and, consequently, a stronger perturbation of the former SS, therefore the convincing IS-SS decoupling is already reached for the 12-layer slab.

The overlap between IS and molecular state wave functions depends on the substrate-adsorbate vertical separation. For the largest distance, the accumulation of the IS charge density on the molecular ML has a minimal value, but it increases as the adsorbate approaches the substrate. The shape of the IS charge density localized on the Tc molecule is insensitive to the adsorption distance, and there is no hybridization of the IS with the unoccupied former LUMO of Tc [see Fig. 4(c)] though they have close energies at the $\bar{\Gamma}$ point. On the contrary, the former LUMO of the aforementioned systems with strong substrate-adsorbate interaction is partially occupied and the
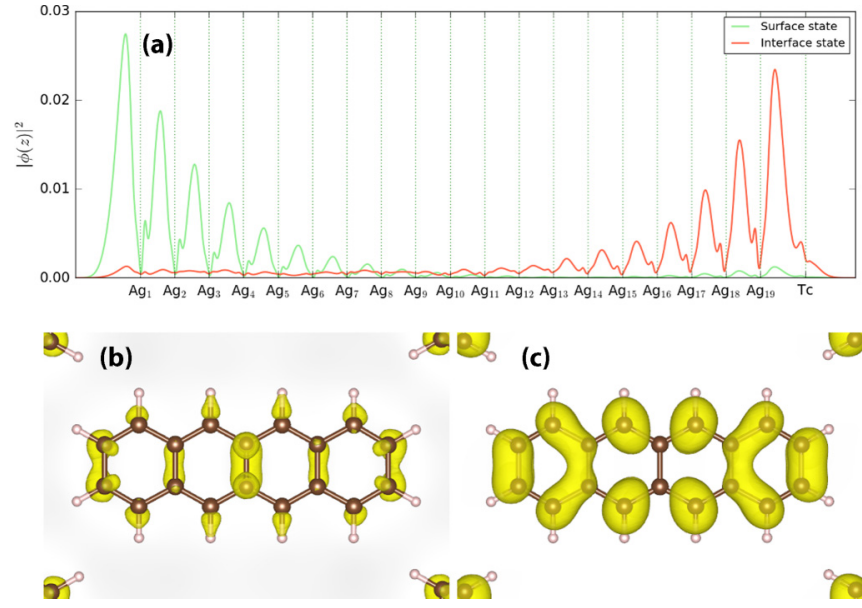

FIG. 4. (a) Charge density distribution averaged over the spatial $x y$ coordinates within the unit cell of the $\gamma-\mathrm{Tc} / \operatorname{Ag}(111)$ for the bridge $\left(\Gamma_{2}\right)$ adsorption geometry. Model 2 with optB88-vdW functional were exploited. The interface (red) and clean-side surface state (green) of the 19-layer slab at the $\bar{\Gamma}$ point are presented as functions of $z$. (b) Charge-density isosurfaces of the IS and (c) the former LUMO.

shape of the IS in the molecular region resembles that of the LUMO of the free molecule [46].

Another factor affecting the magnitude of the SS transformation is the adsorbate coverage or the density of carbon atoms in the surface unit cell [19]. The $\alpha$-phase calculations illustrate the influence of the increased coverage by $\sim 18 \%$; the IS energy is increased by $40 \mathrm{meV}$ in comparison with the the $\gamma$-phase calculations within model 2 and with the 12-layer slab (see Table III). Under the assumption that the dependence of the IS energy on the number of silver layers will be the same as for $\gamma$-Tc/Ag(111) we can conclude that the converged value of the SS energy upshift due to adsorption of Tc monolayer on $\operatorname{Ag}(111)$ in $\alpha$ phase will be about $160 \mathrm{meV}$. The available STS (scanning tunneling spectroscopic) data [24] of $\alpha$-Tc/Ag(111) provides the SS-IS energy difference of $220 \mathrm{meV}$, which is well described by our optB88-vdW calculation.

\section{CONCLUSION}

We performed the theoretical study of the $\mathrm{Tc} / \mathrm{Ag}(111)$ metal-organic interface by means of DFT calculations with localized basis. We focused on the simultaneous description of the structural and electronic properties on equal footings. The optB88-vdW version of the vdW-DF2 family of exchangecorrelation functionals especially optimized for solids was used as well as the conventional GGA-PBE approximation.

The bridge and hollow adsorption sites were determined as the most stable geometries of $\gamma$-Tc/Ag(111) irrespective to the approximation chosen for the exchange-correlation functional, but vdW contribution constitutes the decisive part of the substrate-adsorbate interaction. The inclusion of the long-range dispersive forces into the calculations provides close agreement of the binding energy with the available experimental data [27] and, therefore, better description of the adsorption geometry, whereas the conventional GGA-PBE scheme underestimates the equilibrium adsorption distance. 
We suggested and tested three models of handling the substrate in the metal-organic hybrid interface under study in order to overcome the controversial trends of its bulk and surface electronic properties in the case of localized-basis-set description. We obtained the best result within model 2, where only the silver atoms on the slab surfaces are described by long-range basis orbitals, while the internal (bulk) silver atoms are characterized by rather short orbitals providing adequate bulk properties. In our case of the weakly bound adsorbate, the corrugation of the substrate is small and model 1 with the fixed substrate provides a good description as well. But when considering chemisorbed systems, like PTCDA/Ag(111), the stronger substrate-adsorbate interaction gives rise to the larger substrate corrugation and the possibility for the substrate to be relaxed becomes quite important.

We showed that a quite large lattice constant of the substrate (model 3 ) results in noticeable changes in adsorption geometry and, consequently, leads to inadequate description of the electronic structure. Moreover, the absolute position of the interface state (or surface state in case of pristine surface) is systematically shifted upward with increasing the substrate lattice constant. The difference between the interface and surface state energies is tolerant to the substrate characteristics, it is rather sensitive to the adsorption distance of the adsorbate. The interface states of both phases are fully unoccupied, and, because of the enlarged density of Tc molecules in the $\alpha$ phase, the IS energy is higher than in the $\gamma$ phase. The calculations with the OptB88-vdW functional give not only good values for the binding energy, but the IS energy is in fairly close agreement with the experimental measurements [24].

\section{ACKNOWLEDGMENTS}

This work is a project of the SFB 1083 "Structure and Dynamics of Internal Interfaces" funded by the Deutsche Forschungsgemeinschaft (DFG). We acknowledge partial support from the University of Basque Country UPV/EHU (IT-756-13), the Departamento de Educación del Gobierno Vasco, The Tomsk State University Academic D.I. Mendeleev Fund Program (Grant No. 8.1.05.2015), the Spanish Ministry of Economy and Competitiveness MINECO (Grant No. FIS2013-48286-C2-1-P), and Saint Petersburg State University (Project No. 15.61.202.2015).
[1] C. Dimitrakopoulos and P. Malenfant, Adv. Mater. 14, 99 (2002).

[2] H. Hoppe and N. S. Sariciftci, J. Mater. Res. 19, 1924 (2004).

[3] Y. Shen, A. R. Hosseini, M. H. Wong, and G. G. Malliaras, Chem. Phys. Chem 5, 16 (2004).

[4] Edited by N. Koch, U. Ueno, and A. Wee, The Molecule-Metal Interface (Wiley-VCH, Weinheim, 2013).

[5] N. Koch, J. Phys.: Condens. Matter 20, 184008 (2008).

[6] F. Tautz, Prog. Surf. Sci. 82, 479 (2007).

[7] R. Temirov, S. Soubatch, A. Luican, and F. S. Tautz, Nature (London) 444, 350 (2006).

[8] C. H. Schwalb, S. Sachs, M. Marks, A. Schöll, F. Reinert, E. Umbach, and U. Höfer, Phys. Rev. Lett. 101, 146801 (2008).

[9] N. L. Zaitsev, I. A. Nechaev, and E. V. Chulkov, J. Exp. Theor. Phys. 110, 114 (2010).

[10] M. Marks, A. Schöll, and U. Höfer, J. Electron Spectrosc. Relat. Phenom. 195, 263 (2014).

[11] A. Ferretti, C. Baldacchini, A. Calzolari, R. Di Felice, A. Ruini, E. Molinari, and M. G. Betti, Phys. Rev. Lett. 99, 046802 (2007).

[12] N. Gonzalez-Lakunza, I. Fernández-Torrente, K. J. Franke, N. Lorente, A. Arnau, and J. I. Pascual, Phys. Rev. Lett. 100, 156805 (2008).

[13] J. Ziroff, F. Forster, A. Schöll, P. Puschnig, and F. Reinert, Phys. Rev. Lett. 104, 233004 (2010).

[14] M. Marks, N. L. Zaitsev, B. Schmidt, C. H. Schwalb, A. Schöll, I. A. Nechaev, P. M. Echenique, E. V. Chulkov, and U. Höfer, Phys. Rev. B 84, 081301 (2011).

[15] M. C. E. Galbraith, M. Marks, R. Tonner, and U. Höfer, J. Phys. Chem. Lett. 5, 50 (2014).

[16] B. W. Caplins, D. E. Suich, A. J. Shearer, and C. B. Harris, J. Phys. Chem. Lett. 5, 1679 (2014).

[17] A. Schäfer, I. L. Shumay, M. Wiets, M. Weinelt, T. Fauster, E. V. Chulkov, V. M. Silkin, and P. M. Echenique, Phys. Rev. B 61, 13159 (2000).
[18] M. S. Dyer and M. Persson, New J. Phys. 12, 063014 (2010).

[19] N. L. Zaitsev, I. A. Nechaev, P. M. Echenique, and E. V. Chulkov, Phys. Rev. B 85, 115301 (2012).

[20] E. V. Chulkov, V. M. Silkin, and M. Machado, Surf. Sci. 482485, 693 (2001).

[21] M. Willenbockel, D. Lüftner, B. Stadtmüller, G. Koller, C. Kumpf, S. Soubatch, P. Puschnig, M. G. Ramsey, and F. S. Tautz, Phys. Chem. Chem. Phys. 17, 1530 (2015).

[22] R. W. I. d. Boer, T. M. Klapwijk, and A. F. Morpurgo, Appl. Phys. Lett. 83, 4345 (2003).

[23] C. R. Newman, R. J. Chesterfield, J. A. Merlo, and C. D. Frisbie, Appl. Phys. Lett. 85, 422 (2004).

[24] S. Soubatch, I. Kröger, C. Kumpf, and F. S. Tautz, Phys. Rev. B 84, 195440 (2011).

[25] T. Sueyoshi, M. Willenbockel, M. Naboka, A. Nefedov, S. Soubatch, C. Wöll, and F. S. Tautz, J. Phys. Chem. C 117, 9212 (2013).

[26] A. Langner, A. Hauschild, S. Fahrenholz, and M. Sokolowski, Surf. Sci. 574, 153 (2005).

[27] G. Gonella, H.-L. Dai, and T. Rockey, J. Phys. Chem. C 112, 4696 (2008).

[28] S. García-Gil, A. García, N. Lorente, and P. Ordejón, Phys. Rev. B 79, 075441 (2009).

[29] P. Ordejón, E. Artacho, and J. M. Soler, Phys. Rev. B 53, R10441(R) (1996).

[30] J. M. Soler, E. Artacho, J. D. Gale, A. García, J. Junquera, P. Ordejón, and D. Sánchez-Portal, J. Phys.: Condens. Matter 14, 2745 (2002).

[31] N. Troullier and J. L. Martins, Phys. Rev. B 43, 1993 (1991).

[32] L. Kleinman and D. M. Bylander, Phys. Rev. Lett. 48, 1425 (1982).

[33] J. P. Perdew, K. Burke, and M. Ernzerhof, Phys. Rev. Lett. 77, 3865 (1996). 
[34] J. Klimeš, D. R. Bowler, and A. Michaelides, J. Phys.: Condens. Matter 22, 022201 (2010).

[35] J. Klimeš, D. R. Bowler, and A. Michaelides, Phys. Rev. B 83, 195131 (2011).

[36] K. Lee and J. Yu, Surf. Sci. 589, 8 (2005).

[37] L. Buimaga-Iarinca and C. Morari, Theor. Chem. Acc. 133, 1502 (2014).

[38] D. D. Johnson, Phys. Rev. B 38, 12807 (1988).

[39] T. Ozaki, H. Kino, J. Yu, M. Han, N. Kobayashi, M. Ohfuti, F. Ishii, T. Ohwaki, H. Weng, and K. Terakura, http://openmxsquare.org/.

[40] T. Ozaki, Phys. Rev. B 67, 155108 (2003).

[41] T. Ozaki and H. Kino, Phys. Rev. B 69, 195113 (2004).

[42] T. Ozaki and H. Kino, Phys. Rev. B 72, 045121 (2005).

[43] S. Boys and F. Bernardi, Mol. Phys. 19, 553 (1970).

[44] Y. Tao, H. Mao, H. Zhang, and P. He, Surf. Sci. 641, 135 (2015).
[45] G. Witte, S. Lukas, P. S. Bagus, and C. Wöll, Appl. Phys. Lett. 87, 263502 (2005).

[46] L. Romaner, D. Nabok, P. Puschnig, E. Zojer, and C. AmbroschDraxl, New J. Phys. 11, 053010 (2009).

[47] P. C. Rusu, G. Giovannetti, C. Weijtens, R. Coehoorn, and G. Brocks, Phys. Rev. B 81, 125403 (2010).

[48] K. H. Frank, P. Yannoulis, R. Dudde, and E. E. Koch, J. Chem. Phys. 89, 7569 (1988)

[49] J. B. Neaton, M. S. Hybertsen, and S. G. Louie, Phys. Rev. Lett. 97, 216405 (2006)

[50] D. A. Egger, Z.-F. Liu, J. B. Neaton, and L. Kronik, Nano Lett. 15, 2448 (2015)

[51] G. Li, T. Rangel, Z.-F. Liu, V. R. Cooper, and J. B. Neaton, Phys. Rev. B 93, 125429 (2016).

[52] A. Scheybal, K. Müller, R. Bertschinger, M. Wahl, A. Bendounan, P. Aebi, and T. A. Jung, Phys. Rev. B 79, 115406 (2009). 